\title{
Identification of key attributes of social media influencers: An exploratory study
}

\author{
Chung-Wha Ki ${ }^{1}$, Leslie Cuevas ${ }^{2}$, Heejin Lim ${ }^{2}$ \\ The Hong Kong Polytechnic University ${ }^{1}$, Hong Kong; University of Tennessee ${ }^{2}$, USA
}

Keywords: Social media, Social media influencers (SMIs), Influencer marketing, Focus group

Problem \& Purpose Statement. Influencer marketing, the practice of partnering with social media influencers (hereafter abbreviated as SMIs) to promote or endorse brands' products or/and services, is attracting increasing attention from both academic researchers and marketing practitioners. SMIs are a new type of third-party endorsers who have amassed a solid base of followers on social media by publicly sharing their personal lives, experiences, and opinions through the creation of social media contents. Such public sharings of SMIs' daily lives make consumers view them as more accessible, authentic, and credible than mainstream celebrities (De Veirman, Cauberghe, \& Hudders, 2017); In a similar vein, a message, when communicated by a SMI, is viewed as more authentic than when it is put forward by a celebrity. This makes brands approach SMIs increasingly, asking SMIs to create branded contents and promote those contents both to their own audience and the brands' target consumers (Audrezet, de Kerviler, \& Moulard, 2018). Despite the growing demand for influencer marketing, little effort has been made to understand the genuine factors that affect consumers' perception of SMIs and enable them to exert more influence on audiences. Whilst previous studies in the advertising research stream has documented distinct characteristics that make certain celebrity endorsers more persuasive, such as credibility, attractiveness (Simmers, Damron-Martinez, \& Haytko, 2009), autonomy and competence (Thomson, 2006), little is known about key attributes that enable "SMIs" to generate sizable followers and exert persuasive power. This study fills this void in the literature.

Literature Review. Although extant literature has proposed some drivers that make certain SMIs more suitable for influencer marketing, much has focused on the peripheral traits of SMIs. For instance, Evans, Phua, Lim, and Jun (2017) demonstrated the effects SMIs' disclosure messages ('SP', 'Sponsored', and 'Paid Ad') have on consumers' ad recognition and purchase intention. Braatz's (2017) empirical research validated the effects message sidedness (one-sided vs. twosided) and product depiction (abstract vs. context-based) have on consumers' attitude towards the promotional posts and product liking. Furthermore, De Veirman et al. (2017) experimentally demonstrated the effect SMIs' number of followers has on consumer attitudes (influencer likability and brand attitude); SMIs with a large number of followers was found to be more likable by consumers than SMIs with less followers. However, this effect was moderated by SMIs' number of followees. The ratio of SMIs' followers versus their followees was found to be important in consumers' likeability toward the SMIs. Although a SMI amassed a large number of followers, when s/he had a relatively small number of followees, consumers exhibited negative likability toward her/him. These findings indicate that SMIs with a high number of followers may not necessarily mean they are influential, which implies the need for discovering more principal traits that allow SMIs to amass followers in the first place.

Page 1 of 3

Published under a Creative Commons Attribution License (https://creativecommons.org/licenses/by/4.0/), which permits unrestricted use, distribution, and reproduction in any medium, provided the original work is properly cited.

ITAA Proceedings, \#76 - https://itaaonline.org 
Method. To gain more in-depth insights as to why consumers consider certain SMIs as more likable and followable than others, we conducted a focus group interview. In doing so, we selected Instagram as a target social media platform because of its dominant popularity for influencer marketing (Williams, 2018). Following the suggestion of Nagle and Williams (2013), we invited 11 informants to join our focus group. Because women use and engage in imagebased social media more than men, all participants were female students (Newberry, 2019). These female students were enrolled in retail and consumer sciences courses at one of the major universities in the U.S., had their own account on Instagram, and also followed at least one SMI on Instagram. The focus group was held for about 40 minutes. The focus group was conducted with the attendance of one moderator, who prepared the discussion guide to make sure the session covered all subjects of interest, and another observer, who took notes of the discussion (Nagle \& Williams, 2013). Also, the focus group included open-ended questions to promote discussion, but avoided yes-no questions that would limit discussion (Nagle \& Williams, 2013). The focus-group interview was semi-structured and guided by the following questions: (1) Why do you like and follow your choice of SMIs? and (2) What aspects or/and qualities of your choice of SMIs attract and influence you? We analyzed the discussion immediately after the focus group closure (Nagle \& Williams, 2013).

Findings. Our data analysis revealed six distinct characteristics that determine the brand equity of social media influencers: (1) Authenticity, (2) competence, (3) relatability, (4) physical attractiveness, (5) ideality/paragon, and (6) ability to create quality content. Example descriptors of SMIs' authenticity include being realistic, unique, genuine, honest; participants' perception of SMI's competence was described as confident, charismatic, and successful, and led participants to report this as an important factor that connects them to the SMI; physical attractiveness was another important factor that makes participants stay connected to the SMI; the majority of participants stated the importance of SMIs' being a paragon of their desirable lifestyle such as being a role model and fostering inspirations; lastly, participants also reported that the quality of content that SMIs share on their Instagram is important, including both verbal and visual content. Additionally, our data analysis revealed that without direct advertising of a product, the exposure to SMIs' Instagram content has a persuasive power to alter followers' consumption patterns. The following excerpt shows an example: "I don't usually wear makeup as you can see, but I thought about it after I saw a makeup video of an Instagram influencer who wore shades of pink lipstick. After then, I thought maybe I should wear makeup."

Discussion. Our findings provide implications for both theory and practice. Our findings contribute to the SMI literature by identifying six core qualities (i.e., authenticity, competence, relatability, physical attractiveness, ideality/paragon, and ability to create quality content) that grant SMIs the ability to exert influence over consumers. Whilst some of SMIs' key attributes were found consistent with previous study findings in the context of celebrity endorsers (e.g., source attractiveness, competence, ideality/paragon), our findings also documented a set of unique characteristics of SMIs, distinct from those of celebrities, that consumers take into serious consideration such as authenticity, relatability, and content curation ability. The relatability

Published under a Creative Commons Attribution License (https://creativecommons.org/licenses/by/4.0/), which permits unrestricted use, distribution, and reproduction in any medium, provided the original work is properly cited.

ITAA Proceedings, \#76 - https://itaaonline.org 
attribute of SMIs implies that one of biggest distinctions of influencer marketing from celebrity endorsement may lie in community-based interactions and connections with followers (Geppert, n.d.). Furthermore, interestingly, the content curation ability of SMIs implies that consumers perceive SMIs not only as information sources but also as role models who inspire through their lifestyle choices and competent curation. Our findings also provide guidance for marketing practitioners. In developing and executing successful influencer marketing campaigns, it is essential for marketing practitioners to consider the deep connections between SMIs and their followers. To segment and partner with the so-called right SMIs, we recommend brands marketers to evaluate SMIs' content rather than using the number of followers as a relevant and significant measure. Specifically, the key in collaborating with SMIs seems to be linked to the six attributes, through which SMIs largely translate personality, lifestyle, and taste via their social media content and ultimately foster great inspiration.

$\underline{\text { References }}$

Audrezet, A., de Kerviler, G., \& Moulard, J. G. (2018). Authenticity under threat: When social media influencers need to go beyond self-presentation. Journal of Business Research, 113.

Braatz, L. (2017). \#Influencer marketing on Instagram: Consumer responses towards promotional posts: The effects of message sidedness and product depiction. University of Twente, Enschede, Netherlands.

De Veirman, M., Cauberghe, V., \& Hudders, L. (2017). Marketing through Instagram influencers: the impact of number of followers and product divergence on brand attitude. International Journal of Advertising, 36(5), 798-828.

Evans, N. J., Phua, J., Lim, J., \& Jun, H. (2017). Disclosing Instagram influencer advertising: The effects of disclosure language on advertising recognition, attitudes, and behavioral intent. Journal of Interactive Advertising, 17(2), 138-149.

Geppert, G. (n.d.). How influence marketing differs from celebrity endorsement. Retrieved March 28, 2019, from https://tinyurl.com/tsd2jp2

Nagle, B., \& Williams, N. (2013). Methodology brief: Introduction to focus groups. Center for Assessment, Planning and Accountability. Retrieved from https://tinyurl.com/rdom9rs

Newberry, C. (2019, March 5). 130+ social media statistics that matter to marketers in 2019. Retrieved March 29, 2019, from https://tinyurl.com/tux49ht

Simmers, C. S., Damron-Martinez, D., \& Haytko, D. L. (2009). Examining the effectiveness of athlete celebrity endorser characteristics and product brand type: The endorser expertise continuum. Journal of Applied Sport Management, 1(1), 52-64.

Thomson, M. (2006). Human brands: Investigating antecedents to consumers' strong attachments to celebrities. Journal of Marketing, 70(3), 104-119.

Williams, R. (2018, July 18). Study: Instagram leads as influencer marketing platform. Retrieved March 28, 2019, from https://tinyurl.com/tbv66kf

Page 3 of 3

(c) 2019 The author(s). Published under a Creative Commons Attribution License

(https://creativecommons.org/licenses/by/4.0/), which permits unrestricted use, distribution, and reproduction in any medium, provided the original work is properly cited.

ITAA Proceedings, \#76 - https: //itaaonline.org 